\title{
STRAIN-DRIVEN GENERATIVE DESIGN FRAMEWORK COUPLED WITH A MIMETIC METAMATERIAL: A PROCESS TOWARDS MECHANICAL AND SHAPE ADAPTATION TO OBSERVED STRUCTURES AND FUNCTIONALITIES
}

\author{
Hugo García Modet ${ }^{1}$, Luis Saucedo Mora ${ }^{1,2}$, Miguel Ángel Sanz Gómez ${ }^{1}$ and Francisco \\ Javier Montans ${ }^{1,3}$ \\ ${ }^{1}$ Universidad Politécnica de Madrid \\ ETS de Ingeniería Aeronáutica y del Espacio, Pza Cardenal Cisneros 3, 28040 Madrid \\ 2 Department of Materials, University of Oxford \\ Parks Road, Oxford, OX 1, 3PJ, UK \\ ${ }^{3}$ Department of Mechanical and Aerospace Engineering, Herbert Wertheim College of Engineering \\ University of Florida, FL 32611, USA
}

Key words: Topology Optimization, Generative Design, Metamaterials, FEM

\begin{abstract}
Topology optimization has undergone tremendous development since its introduction by Bendsøe and Kikuci in 1988, especially in recent years, due to its involvement in revolutionary generative design techniques. This paper aims to lay the foundations of a generative design methodology powered by an alternative approach to the well-known density methods. Based on finite element analysis, the objective is to develop an optimization algorithm with the Young modulus of the elements as design variables. That way, while previous studies have focused on void/solid distributions, this study searches for a distribution of different $E$ values that could be manufactured due to progress in metamaterials and additive manufacturing. A mimetic metamaterial was also developed to be coupled with the topological optimization, but will not be included in this paper.

To assess the optimization algorithm, several analyses have been carried out under different load and boundary conditions. The outcome shows correlation with our initial hypothesis: elements under higher strains increase their stiffness value, while the opposite occurs for those under minor stresses. Consequently, the results present a structure with a Young modulus distribution that optimizes the strain energy, and therefore, reduces the displacements.
\end{abstract}

\section{INTRODUCTION}

This sections intends to introduce the concept of topology optimization and its different approaches, in order to understand the context and theoretical framework of our method. Therefore, most of the information included in this section has been gathered from the work of previous authors and synthesized, in order to obtain an overall idea. For deeper information, the readers are referred to papers [10], [6] and [11], in addition to the well-known monograph on topology optimization by Sigmund and Bendsoe [4]. 
As Sigmund perfectly describes, the aim of topology optimization is to give answers to a critical engineering question: how to place material within a prescribed design domain in order to obtain the best structural performance under certain loads and boundary conditions [10]. Using a general formulation based on linear static finite element analysis [10], the topology optimization problem can be written as a typical optimization problem:

$$
\begin{array}{ll}
\text { minimize: } & F=F(\mathbf{u}(\rho), \rho)=\int_{\Omega} f(\mathbf{u}(\rho), \rho) d V \\
\text { subject to: } & G_{i}(\mathbf{u}(\rho), \rho) \leq 0, i=1, \ldots, M \\
& \rho(\mathbf{x})=0 \text { or } 1, \forall \mathbf{x} \in \Omega
\end{array}
$$

The mathematical statement (1) represents the minimization of an objective function $F$, subject to $M$ different constraints $G_{i}$. The material distribution is described by the design variables, commonly represented as densities, $\rho$. These densities can take either value 0 (void) or 1 (solid material) at any point in the design domain $\Omega$. Note that manufacturing procedures and design criteria can introduce additional constraints to the problem.

Those design variables which satisfy the constraints are referred as feasible domain. On the other hand, the term unfeasible domain indicates the set of design variables which outrage at least one of them.

\subsection{Element-based methods}

The topology optimization problem is usually solved by discretizing the design domain into a large number of finite elements

$\mathrm{N}$ components of this Consequently, the optin
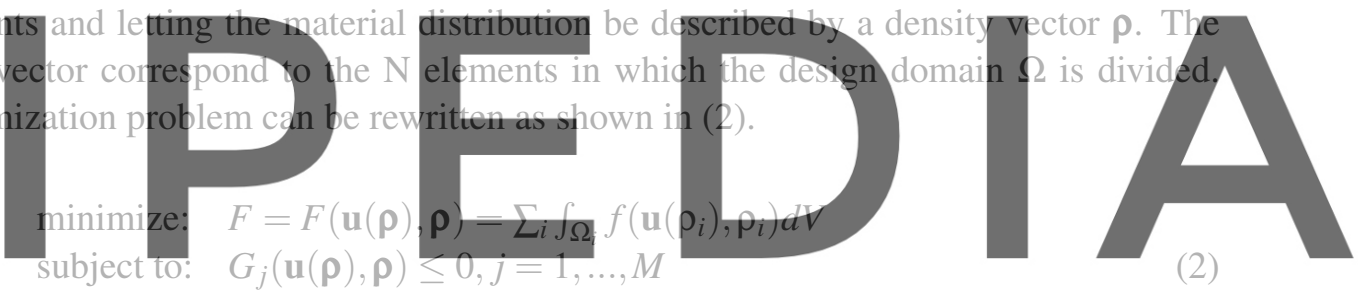

$\rho_{i}=0$ or $1, i=1, \ldots, N$

Register for free at https//www.scipedia.com to download the version without the watermark

Where $\rho$ denotes the design variable vector of length $N$, and $\rho_{i}$ its components. The remaining variables are the same as stated in (1). This formulation establishes that the design variables $p_{i}$ can only take discrete values: 0 or 1 . This constitutes a problem when trying to solve the optimization.

On the other hand, real life optimization is complex and subject to several performance and manufacturing constraints. For that reason, continuous density problems allow each of the components of $\boldsymbol{\rho}$ to take any value between 0 (void) or 1 (solid material). This formulation constitutes the basis of the most successful optimization approaches: density methods.

\subsection{Density approach}

As stated before, these methods imply the discretization of the design domain in a large number of finite elements with the goal of minimizing an objective function. This is achieved by assigning each element a density value between 0 and 1 . The fundamental mathematical statement of a density-based optimization problem [6] may be represented as: 


$$
\begin{array}{cl}
\text { minimize: } & f=f(\mathbf{u}(\boldsymbol{\rho}), \boldsymbol{\rho}) \\
\text { subject to: } & \mathbf{K}(\boldsymbol{\rho}) \mathbf{U}=\mathbf{F} \\
& G_{j}(\mathbf{u}(\boldsymbol{\rho}), \boldsymbol{\rho}) \leq 0, j=1, \ldots, M \\
& 0 \leq \rho_{i} \leq 1, i=1, \ldots, N
\end{array}
$$

For the sake of simplicity, the optimization will be run under linear static conditions, where $f$ is the objective function, typically the strain energy or compliance. Under prescribed forces, the minimization of the compliance means increasing the stiffness of the structure. Then, $\rho$ is the vector of density design variables, $\mathbf{U}$ is the displacement vector, $\mathbf{K}$ is the global stiffness matrix, $\mathbf{F}$ is the force vector and $G_{i}$ represent the problem constraints. Notice that the structural stiffness depends on the density values at element level. The stress energy or compliance is a measure of the structural stiffness, calculated as follows:

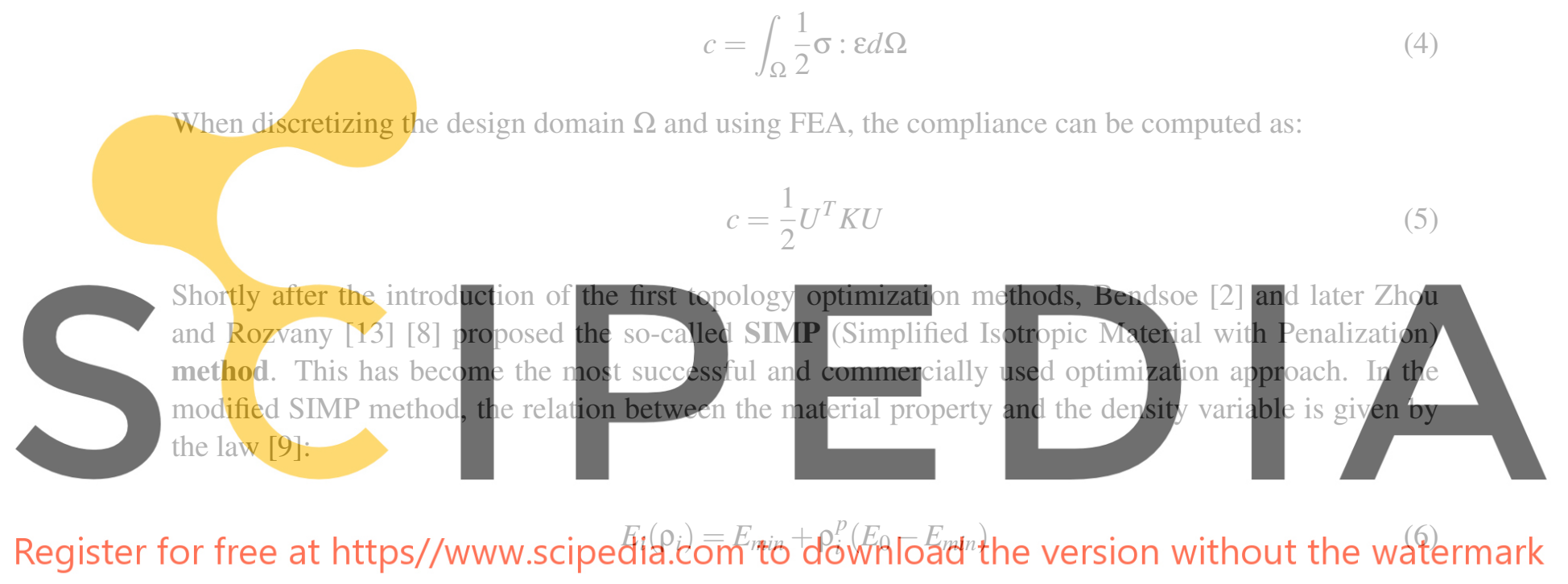

Where $E_{0}$ is the Young's elastic modulus of solid material, $E_{m i n}$ is introduced to avoid numerical instability problems when element density become zero, and $p$ is the penalization parameter used for forcing solutions to suitable void/solid distributions. Nowadays, the simplicity and ease of application of the SIMP method has led to its widespread use in both academic and industrial purposes.

As seen, in the last 30 years since its introduction, topology optimization has become an important field of research, probably driven by the large amount of potential applications and advantages in the preliminary design and optimization phases of products.

Besides its direct impact in the design process, topology optimization has also been a "stepping stone" for the arise of new CAD technologies, such as generative design. This technique takes advantage of cloud computing and machine learning to create hundreds of design options from a set of design requirements (hold areas, cost, weight, material, loads, constraints, performance, manufacturing methods...). Since these results are based on artificial intelligence, in addition to other algorithms, the designs obtained are completely unique and impossible to obtain through traditional methods. 
For all these reasons, generative design and topology optimization are widely considered to be a "game changer" in design processes. Moreover, with the improvements in additive manufacturing in the following years, generative design will inevitably become a standard procedure in product design. This impact will probably be greater in the aerospace industry, due to the savings in weight, fuel consumption and $\mathrm{CO}_{2}$ emissions it involves. Therefore, the aim of this dissertation is to serve as an introduction to the topological optimization and generative design processes that will gain so much relevance in the near future.

This paper is organized as follows. Section 1 has given a brief overview of topology optimization, its recent development and different approaches. Section 2 describes the theoretical basis for our optimization approach, including the hypothesis and governing equations for the finite element analysis, and the explanation of the optimization algorithm. Then, Section 3 presents an example to illustrate the method and show the results. Finally, Section 4 discusses the results and states the conclusions of the research.

\section{THEORETICAL FRAMEWORK}

Traditionally, successful topology optimization approaches have used fictional densities as design variables. However, this work presents an alternative topology optimization approach where no densities are assigned to the elements. Instead, the optimization problem is directly addressed with the elements' Young modulus, $E_{i}$, as design variables. Consequently, the optimized design will present a stiffness layout that will depend on the strain field obtained from the FEA.

These designs, with several Young modulus values, would have been a manufacturing problem years ago,
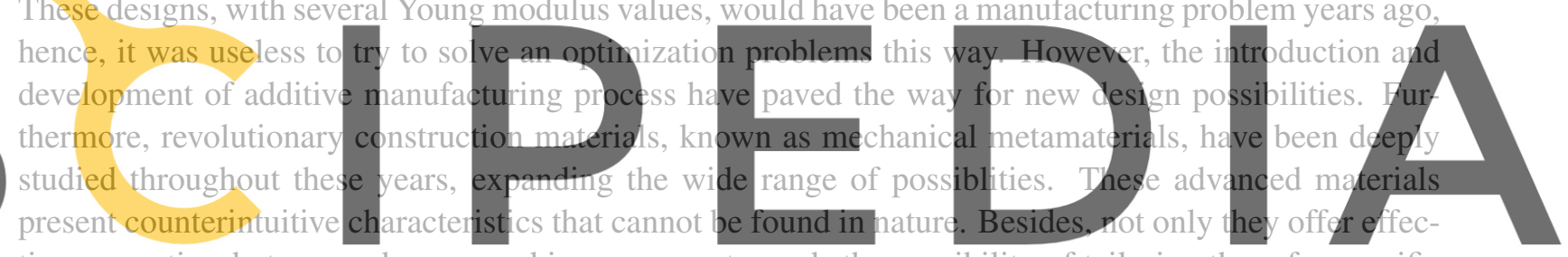

tive properties, but researches are making progress towards the possibility of tailoring them for specific

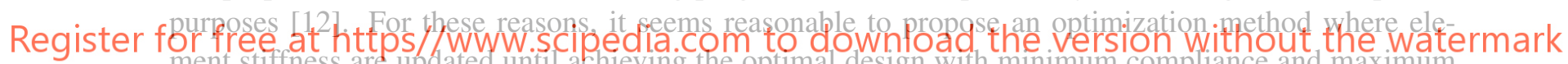
ment stiffness are updated until achieving the optimal design with minimum compliance and maximum stiffness (improved performance).

\subsection{Linear Elasticity}

The optimization presented in this work is element-based and it is inspired by the density approach. Therefore, it is necessary to develop a robust finite-element analysis code capable of computing the displacements and strains that will determine the optimization result. The original FEA code was written by Luis Saucedo Mora.

The optimization will be carried out under the hypothesis of linear elasticity. Hence, the equilibrium equations for a solid body with the domain $\Omega$ are given by [1]:

$$
\begin{array}{ll}
\nabla \cdot \boldsymbol{\sigma}(\mathbf{u})+\mathbf{f}=\mathbf{0} & \text { in } \Omega, \\
\mathbf{u}=\mathbf{0} & \text { on } \Gamma_{D}, \\
\boldsymbol{\sigma}(\mathbf{u}) \mathbf{n}=\overline{\mathbf{t}} & \text { on } \Gamma_{N}
\end{array}
$$


where $\boldsymbol{\sigma}$ is the stress tensor, $\mathbf{u}$ is the displacement vector, $\mathbf{f}$ is the external load vector and $\overline{\mathbf{t}}$ is the prescribed traction on the Neumann boundary $\Gamma_{N}$.The Dirichlet boundary is represented by $\Gamma_{D}$.

The displacement field in a three-dimensional solid is represented by the vector:

$$
\mathbf{u}=[u, v, w]^{T}
$$

where $u, v, w$ are the the displacements of the points in the direction $x, y, z$ of the cartesians axes, respectively.

The strain field, represented by the strain vector $\boldsymbol{\varepsilon}$ is obtained from the displacements derivatives. The formulation used in this work is shown in (9).

$$
\boldsymbol{\varepsilon}=\left[\varepsilon_{x}, \varepsilon_{y}, \varepsilon_{z}, \varepsilon_{x y}, \varepsilon_{y z}, \varepsilon_{x z}\right]^{T}
$$

The stress-strain relationship is expressed for the general case of anisotropic elasticity by a symmetric $6 \times 6$ constitutive matrix with 21 independent parameters, $\mathbf{D}$ :

$$
\sigma=\mathbf{D} \cdot \varepsilon
$$

Assuming isotropic material, the constitutive matrix $\mathbf{D}$ depends only on two parameters: the Young modulus, $E$, and the Poi
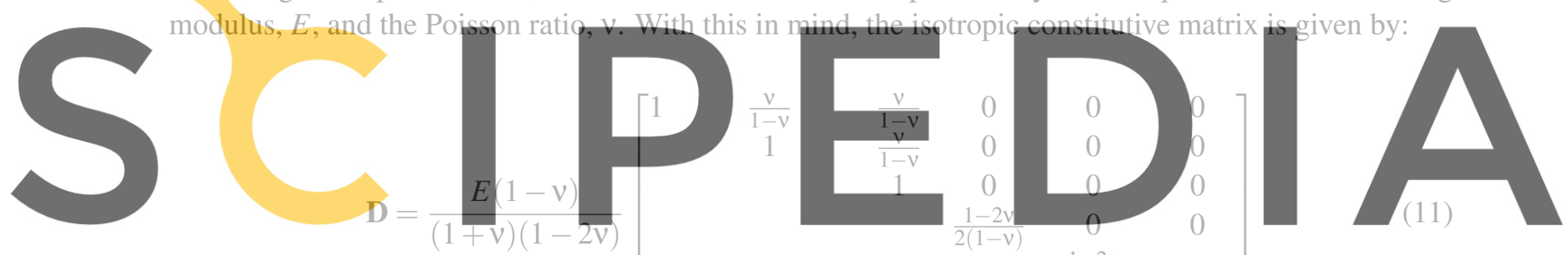

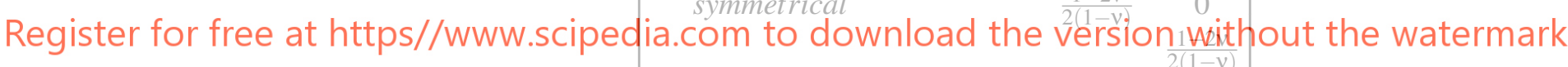

Consequentiy, the stress vector, $\sigma$ results in:

$$
\boldsymbol{\sigma}=\left[\sigma_{x}, \sigma_{y}, \sigma_{z}, \sigma_{x y}, \sigma_{y z}, \sigma_{x z}\right]^{T}
$$

\subsection{Finite Element Formulation}

Once the basic theory for the 3-D analysis has been presented, the next step is to describe the FEM formulation used to run the analysis. In this case, four-noded regular tethaedral elements are going to be used.

The reader is referred to books [7] and [5] for a detailed and complete description regarding the FEA formulation employed in this analysis. 


\subsection{Topology Optimization Approach}

The aim of this paper is to present a new topology optimization approach with no densities assigned to the elements. Instead, the Young modulus of each of them, $E_{i}$, will be updated with the goal of minimizing the strain energy. Once the optimization is completed, a Young modulus distribution dependent on the strain level of the elements will be achieved. This is, those elements under higher strains, will increase their stiffness; and the opposite will happen to those under lower strains. A similar mathematical statement to those of Chapter 1 can be applied to this approach:

$$
\begin{array}{ll}
\text { minimize: } & c=\int_{\Omega} \frac{1}{2} \sigma: \varepsilon d \Omega \\
\text { subject to: } & \mathbf{K}(\mathbf{\rho}) \mathbf{U}=\mathbf{F} \\
& G_{j}(\mathbf{u}(\mathbf{\rho}), \boldsymbol{\rho}) \leq 0, j=1, \ldots, M \\
& 0 \leq E_{i} \leq E_{\max }, i=1, \ldots, N
\end{array}
$$

Where the objective function is the structural compliance, $c$, computed as shown in (5), $G_{j}$ represents additional constraints, $E_{i}$ are the design variables which represent the Young modulus of element $i$, and $E_{\max }$ is the maximum Young modulus value, necessary to achieve the convergence of the method.

In order to describe the optimization algorithm, the expression (4) is developed:
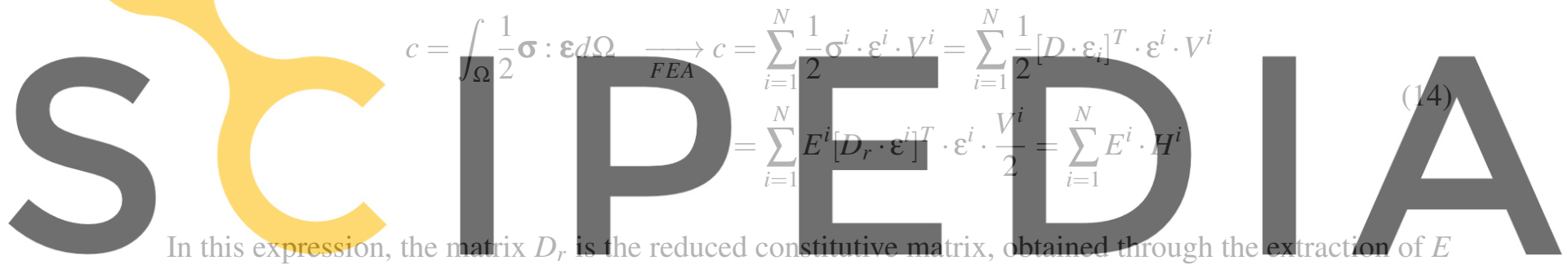

from the matrix (11); $V^{i}$ is the element volume; and the strain parameter of element $i, H^{i}$, is computed as

Register forl

$$
H^{i}=\left[D_{r} \cdot \varepsilon^{i}\right]^{T} \cdot \varepsilon^{i} \cdot \frac{V^{i}}{2}
$$

Hence, this parameter only depends on the element strain field, $\varepsilon^{i}$, since $D_{r}$ and $V^{i}$ are constant values for all the elements of the mesh.

The idea behind our topology optimization approach is to increase the stiffness of those elements with higher $H^{i}$ and to decrease those with lower $H^{i}$. As a result, strains are relaxed and the compliance is minimized. Equation (16a) and constraints (16b) and (16c) are utilized to update the Young modulus.

$$
\begin{gathered}
E_{n+1}^{i}=E_{n}^{i}\left(\frac{H_{i}-\bar{H}}{\sigma_{H_{i}}}+1\right)=E_{n}^{i} \cdot K_{i} \\
K^{i}>0 \\
E_{n+1}^{i} \leq E_{\max }
\end{gathered}
$$


$\bar{H}$ and $\sigma_{H_{i}}$ are the mean value and the standard deviation, respectively. They are computed from all the elements in that optimization step. This means that the further $H_{i}$ is from the others (measured by the mean value), the greater the increase or decrease in the element stiffness. $E_{n+1}^{i}$ represents the Young modulus of element $i$ in iteration $n+1$ and $E_{\max }$ is a parameter used to improved the convergence of the method.

With these results, it is possible to present the algorithm (Algorithm 1) used to update the stiffness of the elements under the relationship (16a).
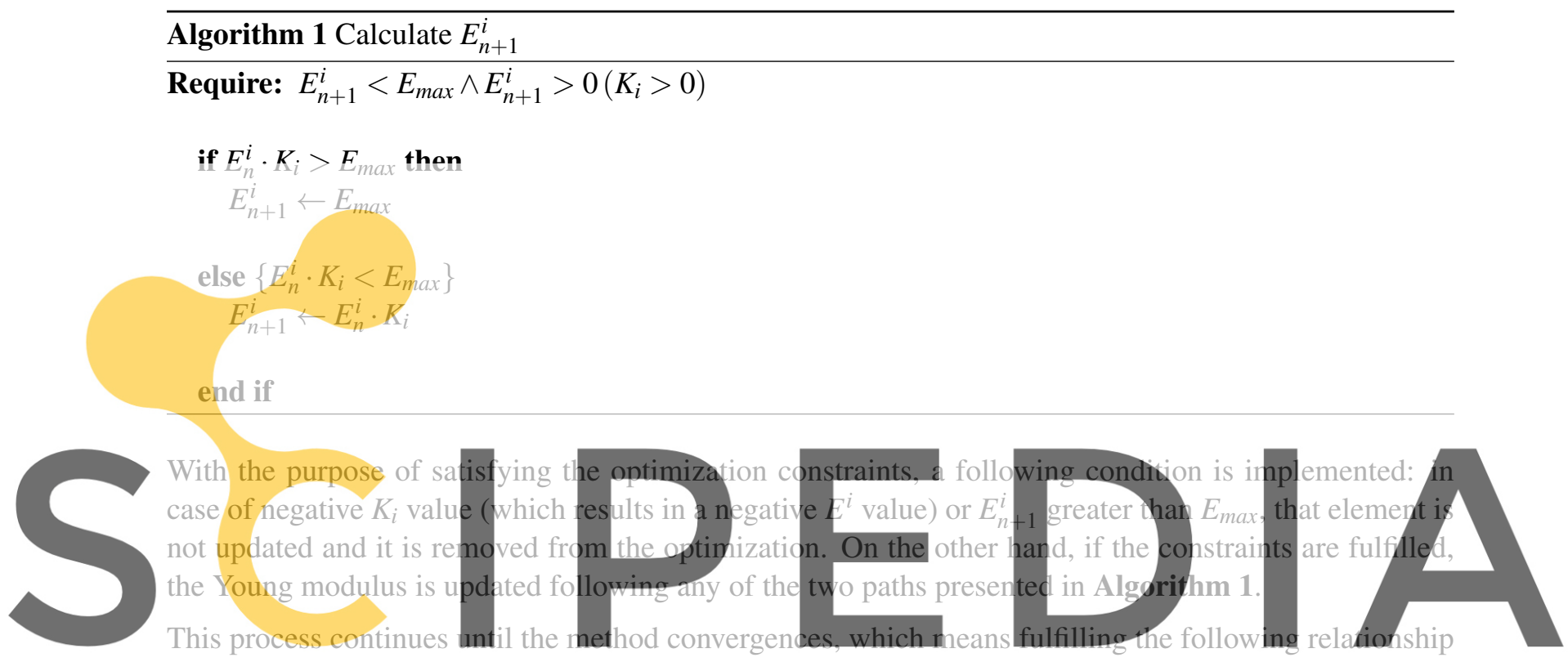

two consecutive times.

Register for free at https//www.scipedia.com to download the version without the watermark

$$
\frac{C_{n}-C_{n-1}}{C_{n-1}}<\text { tol }
$$

where $t o l$ is a parameter chosen when setting the optimization process.

The flowchart in Figure 1 summarizes the optimization loop explained during this section. 


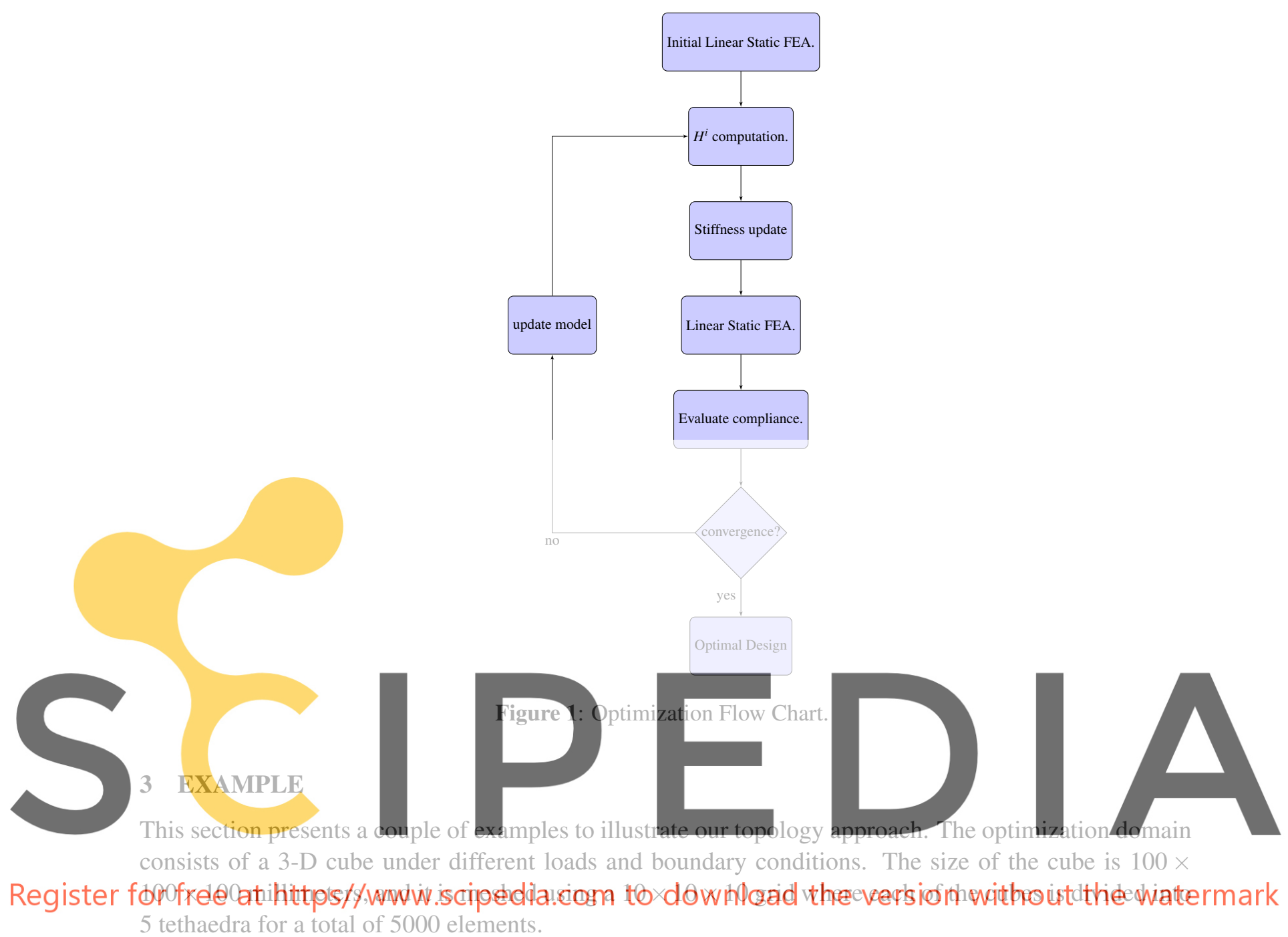

In this example, the loads and boundary conditions are applied aiong a circumference with a $20 \mathrm{~mm}$ radius, as shown in Figure 2(a). The red arrows at the top illustrate the forces, while the blue triangles at the bottom represent the displacement boundary conditions. These values, along with the Poisson ratio $(v)$ and the initial Young modulus $\left(E_{0}\right)$ are given in Table 1.

\begin{tabular}{lcc}
\hline & Value & Direction \\
\hline$E_{0}$ & $1000.0 \mathrm{MPa}$ & - \\
$v$ & 0.3 & - \\
Force & $1000.0 \mathrm{~N}$ & $\mathrm{Z}$ \\
Displacements & 0.0 & All
\end{tabular}

Table 1: Case parameters.

Consequently, we obtain the z-strains field shown in Figure 2(b). This result is key to understand the 
stiffness distribution obtained through the optimization process. As explained in the preceding sections, the basis of our method is the following: elements under higher strains develop greater stiffness values, while those under lower strains reduce theirs. Hence, in this case, a stiffer cylindrical structure must grow inside the cube as a result of the loads and boundary conditions applied.
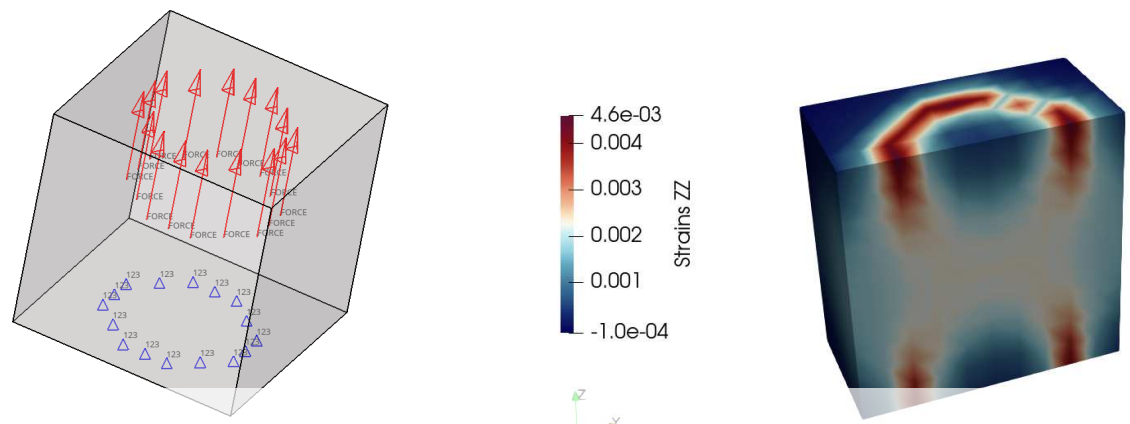

(a)

(b)

Figure 2: Case configuration and FEA (Z-strains)

When running the optimization with a value of $E_{\max }=10000.0 \mathrm{MPa}$ and $t o l=0.005$, the following results are obtained. First, the Young modylus layout is presented in Figure 4(a). For a better understanding of the results, value along the plotted
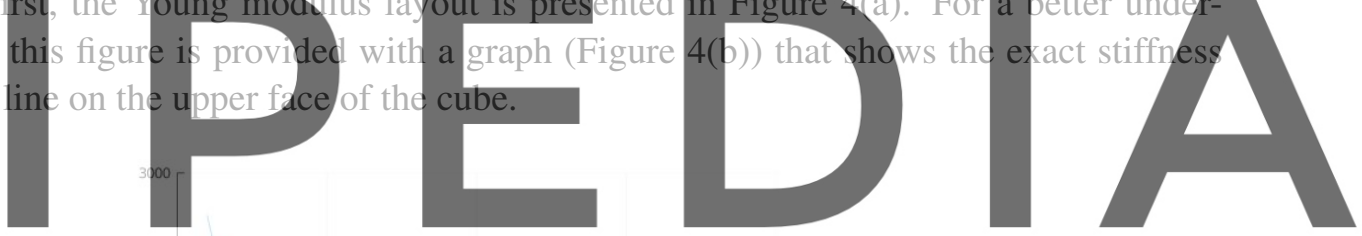

Register for free at https//www.sçipedia.com to download the version without the watermark

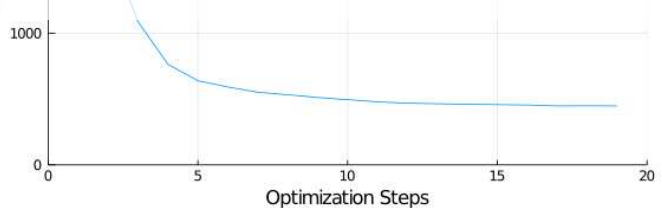

Figure 3: Compliance evolution.

On the other hand, Figure 5 illustrates the topology optimization steps towards the optimal design. They show the generation of a stiffer structure during the optimization process. The blue translucent layer represents a Young modulus of $4000 \mathrm{MPa}$, while the inner brown solid layer corresponds to $6000 \mathrm{MPa}$ (note that the original stiffness was $1000 \mathrm{MPa}$ ). With the purpose of verifying the decrease in the strain energy, Figure 3 illustrates the evolution of the structural compliance. 

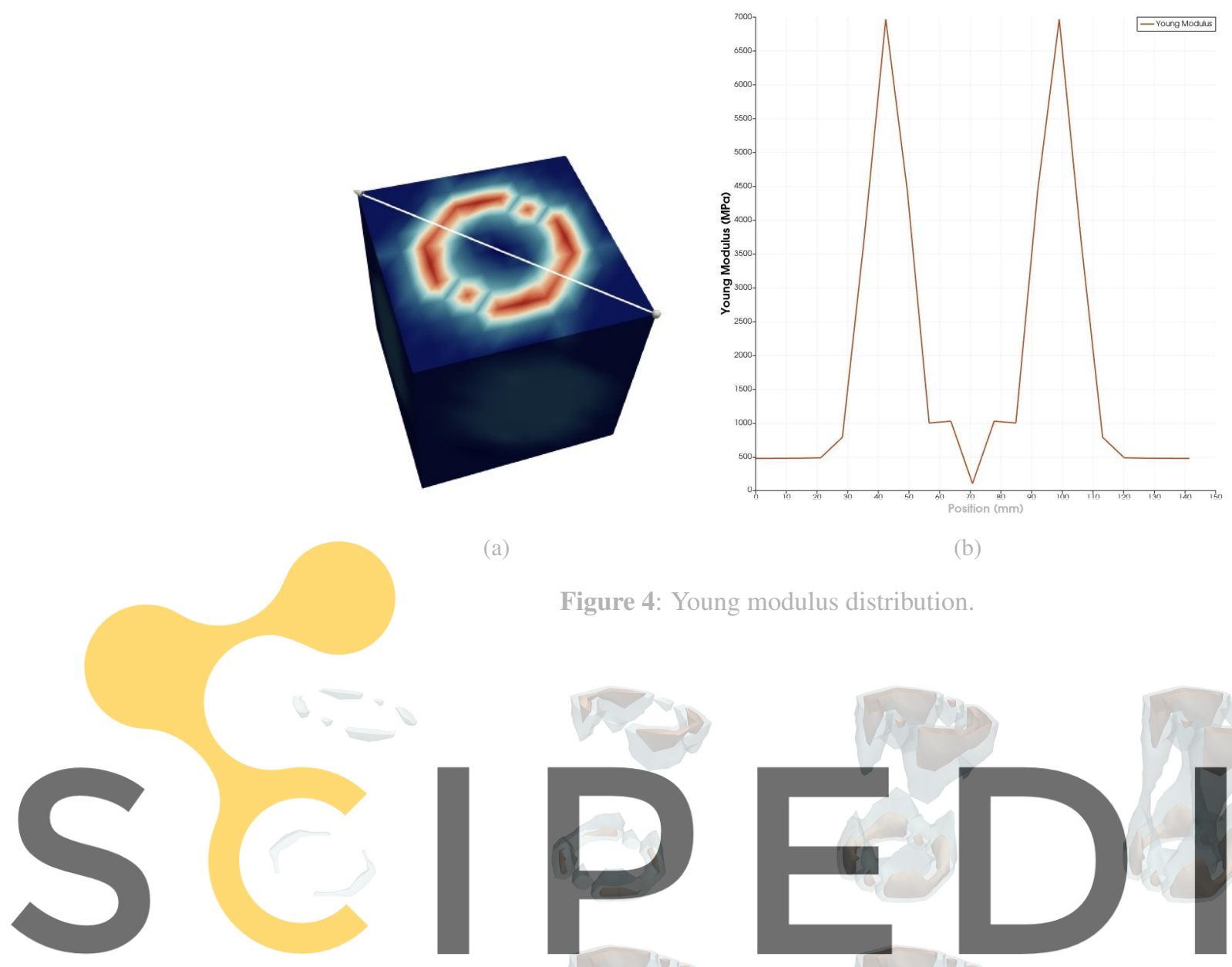

(a)

(b)

Figure 4: Young modulus distribution.
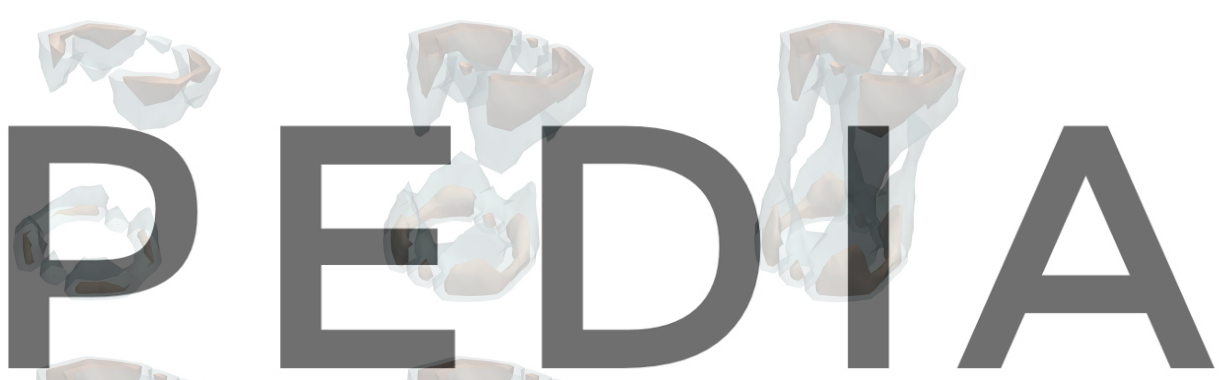

Register for free at https//www.scipedia.com to download the version without the watermark
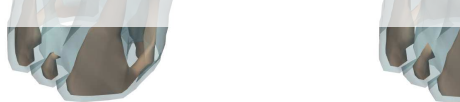

Figure 5: Stiffness evolution during optimization steps.

\section{CONCLUSIONS}

This paper presents the introduction of a generative design methodology powered by an alternative topology optimization approach to the well-known density methods. That way, while previous studies have focused on void/solid distributions, this study searches for a distribution of different $E$ values that could be manufactured due to progress in metamaterials and additive manufacturing. Consequently, the outcome presents a structure with a Young modulus distribution that optimizes the strain energy, and therefore, reduces the displacements.

The presented results corroborate our initial hypothesis: elements with low strains reduce their $E$ value, 
while those suffering greater stresses become stiffer. However, although the results match our expectations, it is worth comparing them with those obtained with a commercial software, for example, HyperWorks (OptiStruct Solver). Therefore, if we observe Figure 6(a), we can appreciate a close similarity between both results. Moreover, Figure 6(b) shows a load case with an important component of shear stress, and again, the optimization result is comparable to that from OptiStruct. Hopefully, we believe that his fact enhances the validity of our model and indicates that our study is on the right track. However, these conclusions should be treated with caution, given that our findings are based on a limited number of cases.

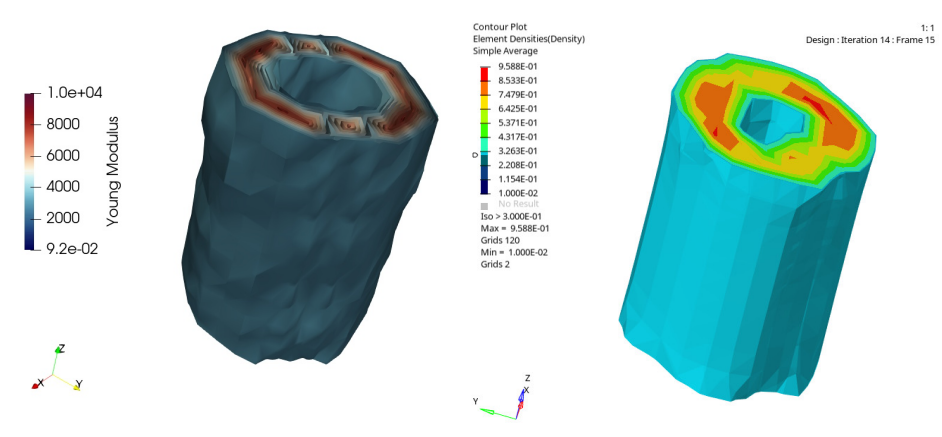

(a)

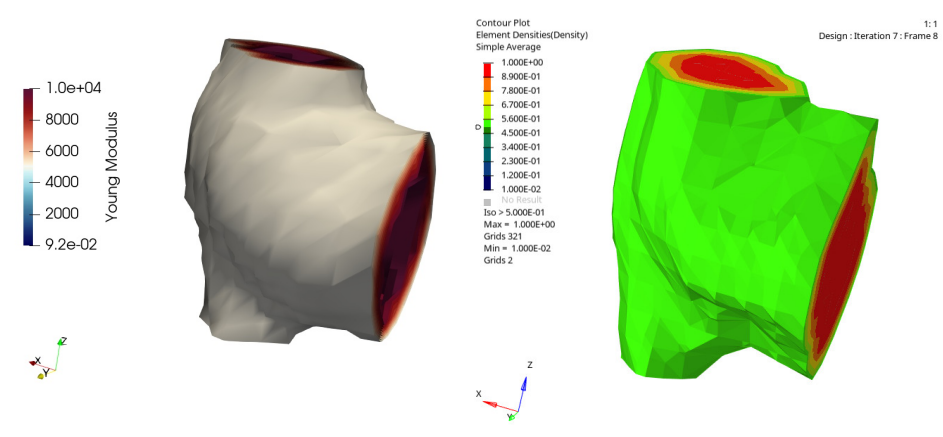

(b)

Figure 6: Optimization results comparison between our code (left) and HyperWorks' OptiStruct (right) for the different load cases. 


\section{REFERENCES}

[1] Kosala Bandara, Thomas Rüberg, and Fehmi Cirak. Shape optimisation with multiresolution subdivision surfaces and immersed finite elements. Computer Methods in Applied Mechanics and Engineering, 300:510-539, 2016.

[2] M. P. Bendsøe. Optimal shape design as a material distribution problem. Structural Optimization, 1(4):193-202, dec 1989.

[3] M. P. Bendsøe and Noboru Kikuchi. Generating optimal topologies in structural design using a homogenization method. Computer Methods in Applied Mechanics and Engineering, 71(2):197224, nov 1988.

[4] M. P. Bendsøe and Ole Sigmund. Topology Optimization. Springer Berlin Heidelberg, Berlin, Heidelberg, 2004.

[5] Eduardo W. Chaves and Roberto Mínguez. Mecánica Computacional en la Ingeniería con Aplicaciones en MATLAB. 012010.

[6] Joshua D. Deaton and Ramana V. Grandhi. A survey of structural and multidisciplinary continuum topology optimization: Post 2000, jan 2014.

[7] E. Oñate. Structural Analysis with the Finite Element Method. Linear Statics, volume 53. 2012.

[8] G. I. N. Rozvany, M. Zhou, and T. Birker. Generalized shape optimization without homogenization. Structural Optimization, 4(3-4):250-252, sep 1992.

[9] Ole Sigmund. Morphology-based black and white filters for topology optimization. Structural and Multidisciplinary Optimization, 33(4-5):401-424, apr 2007.

[10] Ole Sigmund and Kurt Maute. Topology optimization approaches: A comparative review, dec 2013.

[11] Evangelos Tyflopoulos, David Tollnes Flem, Martin Steinert, and Anna Olsen. State of the art of generative design and topology optimization and potential research needs. Technical report, 2018.

[12] Xianglong Yu, Ji Zhou, Haiyi Liang, Zhengyi Jiang, and Lingling Wu. Mechanical metamaterials associated with stiffness, rigidity and compressibility: A brief review, may 2018.

[13] M. Zhou and G. I.N. Rozvany. The COC algorithm, Part II: Topological, geometrical and generalized shape optimization. Computer Methods in Applied Mechanics and Engineering, 89(1-3):309336, aug 1991. 